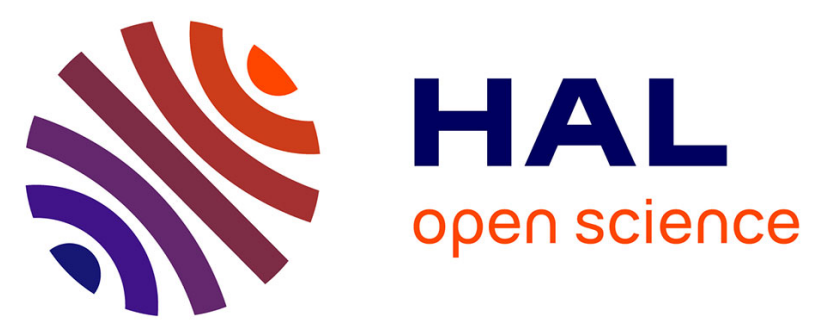

\title{
La Distance à la performance attendue : un indicateur des choix de l'enseignant en fonction du potentiel de chaque élève
}

\author{
Jean-Jacques Maurice, Audrey Murillo
}

\section{To cite this version:}

Jean-Jacques Maurice, Audrey Murillo. La Distance à la performance attendue : un indicateur des choix de l'enseignant en fonction du potentiel de chaque élève. Revue Française de Pédagogie, 2008, 162, pp.67-79. 10.4000/rfp.827 . halshs-03087117

\section{HAL Id: halshs-03087117 \\ https://shs.hal.science/halshs-03087117}

Submitted on 7 Jan 2021

HAL is a multi-disciplinary open access archive for the deposit and dissemination of scientific research documents, whether they are published or not. The documents may come from teaching and research institutions in France or abroad, or from public or private research centers.
L'archive ouverte pluridisciplinaire HAL, est destinée au dépôt et à la diffusion de documents scientifiques de niveau recherche, publiés ou non, émanant des établissements d'enseignement et de recherche français ou étrangers, des laboratoires publics ou privés. 


\title{
La Distance à la Performance Attendue : un indicateur des choix de l'enseignant en fonction du potentiel de chaque élève
}

Jean-Jacques Maurice

Audrey Murillo

\begin{abstract}
Certaines dimensions des pratiques d'enseignement résulteraient d'une adaptation aux contraintes liées à la gestion d'une classe hétérogène. L'étude présentée porte sur des séances de lecture (découverte de texte), au Cours Préparatoire, et s'attache à analyser à la fois les choix de tâches proposées par les enseignants et les potentialités des élèves par rapport à ces tâches. Cet article présente un indicateur, la Distance à la Performance Attendue (DPA), qui mesure pour chaque élève l'écart entre ce qu'il sait faire et ce qu'il faudrait savoir faire pour effectuer la tâche choisie par l'enseignant. Les DPA révèlent que les enseignants sont quotidiennement contraints d'opter pour des niveaux de difficulté de tâches qui conviennent mieux à certains élèves de niveau moyen en début d'année. Alors qu'il existe peu d'enjeu pour les élèves faibles et forts (qui ne changent pas de statut en cours d'année), certains élèves moyens ont l'opportunité de progresser beaucoup plus que d'autres: l'enseignant semble contraint de cibler ses actions sur ce « groupe de pilotage » pour faire évoluer l'ensemble de la classe.
\end{abstract}

\section{NTRODUCTI ON}

Les opportunités d'apprendre à lire, pour chaque élève de cours préparatoire, dépendraient en partie de la succession des contextes éducatifs choisis par l'enseignant. Or, face à une classe hétérogène, il est probablement impossible de choisir, au quotidien, des supports d'apprentissages convenant à tous les élèves. Sur la durée d'une année scolaire, les contraintes de choix pour l'enseignant imposeraient, aux élèves d'une même classe, des opportunités d'apprendre contrastées.

\section{LES DIFFERENTS « GRAI NS D'ANALYSE 》 D'UNE SITUATION EDUCATIVE : UN ECLAI RAGE, MAIS DES ZONES OBSCURES...}

Dans le cadre des recherches s'intéressant aux pratiques effectives des enseignants il semble difficile d'étudier, de façon précise, voire microscopique, l'action d'un enseignant, tout en prenant en compte l'action de chaque élève dans la classe face à un enjeu de savoir précisément identifié. Un tel projet devient encore plus complexe lorsqu'il s'agit d'élargir l'étude au delà d'une observation ponctuelle afin d'approcher l'effet du temps à l'échelle d'une année scolaire.

Quelques exemples caractéristiques, n'ayant pas l'ambition d'une revue de question, montrent que la focalisation d'une recherche sur une dimension de la situation observée impose une révision à la baisse des investigations sur d'autres dimensions.

Ainsi, les didacticiens qui défendent à juste titre le rôle du savoir en jeu dans la relation éducative, s'appuient, par exemple, sur la théorie des situations didactiques de Brousseau (1986), décrivent de façon qualitative le rôle de l'enseignant, mais considèrent, le plus souvent, un élève épistémique alors que la classe observée est hétérogène. 
Des travaux en sciences de l'éducation, étudiant les pratiques enseignantes (Bressoux, Bru, Altet \& Lambert, 1999) décrivent, de façon très précise, le comportement de l'enseignant, ils sont alors contraints d'évoquer seulement l'action de l'élève ou l'influence des savoirs.

Etudier le travail de l'enseignant d'un point de vue théorisé, par exemple à travers les concepts et méthodes utilisés en ergonomie (Amigues, 2003), permet également de montrer la complexité de l'activité enseignante, mais impose de limiter l'étude de celle de l'élève.

Des chercheurs rapprochant sciences cognitives et éducation (Gentaz \& Dessus, 2004) étudient majoritairement la cognition de l'élève pour, dans un deuxième temps, formuler des recommandations concernant ce que l'enseignant devrait faire pour faciliter les apprentissages. Cette pratique prescrite ne dit rien de sa contextualisation effective future.

Notre contribution n'a pas la prétention de construire une méthodologie permettant de résoudre tous les problèmes évoqués ci-dessus. Tout spécialiste d'une des dimensions étudiées en trouvera aisément les limites. Notre méthodologie tente de développer un indicateur qui éclairerait à la fois un aspect de l'activité de l'enseignant (ses décisions de choix du niveau de difficulté d'une tâche) et la conséquence de ce choix en fonction du potentiel de chaque élève de la classe. Cet indicateur (la Distance à la Performance Attendue : écart entre ce qu'il faudrait savoir faire pour effectuer la tâche et ce que chaque élève sait faire ${ }^{1}$ ) est utilisé à plusieurs reprises au cours d'une année scolaire au cours préparatoire afin de donner à voir l'évolution de cette relation : choix de l'enseignant/potentiels hétérogènes des élèves. Nous tenterons de montrer dans cette contribution la portée heuristique de l'indicateur de Distance à la Performance Attendue. En effet, cet indicateur permet de mesurer l'hétérogénéité didactique de la classe, hétérogénéité "créée par le système didactique » (Sarrazy, 2003), due non seulement aux différents potentiels des élèves, mais aussi aux choix des enseignants qui, par les tâches proposées, donnent à voir ces différences de potentiels. La construction de cet indicateur s'appuie sur des travaux antérieurs et des présupposés théoriques.

\section{TRAVAUX ANTERI EURS, PRESUPPOSES THEORI QUES}

Nos travaux antérieurs (Maurice, 1996a, b) ont permis de montrer que l'expérience des enseignants génère des savoir faire qui révèlent à quel point les tâches qu'ils proposent à leurs élèves ont une valeur d'utilité dans leur quotidien. C'est en lien avec ces tâches qu'ils ont développé des habiletés : ainsi ils prévoient, dès lecture d'une tâche, quels élèves vont réussir ou échouer.

La dimension cognitive de l'expérience de l'enseignant ne dépendrait pas uniquement de ce qu'il a appris en formation. Comme tout professionnel, une grande partie de son expérience se construirait dès ses premiers pas dans le métier, par adaptation à un milieu qui lui résiste (selon le constructivisme piagétien évoqué habituellement lorsqu'il s'agit de l'élève). Ses savoirs ou savoir faire ne seraient donc pas uniquement le fruit d'une formation initiale (Maurice, 2006a), ils se transforment et évoluent au fil de l'expérience :

- ils échappent en grande partie aux institutions de formation ;

- ils sont difficiles d'accès pour l'observateur et peu exprimables par l'acteur (ils résistent parfois à ses tentatives réflexives);

- ils sont mobilisés sur des situations proposées quotidiennement aux élèves et ont ainsi, probablement, des conséquences sur l'apprentissage. 
Pour l'enseignant, les tâches proposées aux élèves pourraient être considérées comme des outils visant l'enrôlement et l'apprentissage (Doyle, 1985). Rabardel (1995) précise que lorsqu'un outil est associé à des schèmes (Vergnaud, 1985), il devient instrument ; ainsi ces tâches qui structurent et prolongent la cognition de l'enseignant, lui permettent d'anticiper les performances des élèves (Maurice, 1996a, b). Il les utiliserait comme instruments pour "penser sa classe ", elles seraient intégrées à sa cognition. Il leur déléguerait ainsi une partie de son pouvoir : c'est elles qui, à sa place, peuvent rassurer ou inquiéter, mobiliser ou démobiliser, valoriser ou dévaloriser, etc. Il maintiendrait ainsi indirectement son pouvoir en faisant varier le curseur de la difficulté.

Si l'enseignant, à la lecture d'une tâche, peut anticiper les performances de ses élèves, il s'agit d'identifier, au cours d'une année scolaire, comment il utilise son pouvoir d'anticipation. Nous supposons cependant que sa marge de manœuvre n'est pas pour autant très importante. En effet, (Maurice 2006b) le pouvoir d'enrôlement d'une tâche facilite la conduite de la classe. Certaines tâches, trop faciles ou trop difficiles démobilisent rapidement un trop grand nombre d'élèves qui risquent alors d'échapper au « contrôle » de l'enseignant.

\section{CHOI X METHODOLOGI QUES}

Notre hypothèse : l'enseignant est quotidiennement contraint d'opter pour des niveaux de difficulté de tâches qui conviennent mieux à certains élèves qu'à d'autres :

- certains élèves, plus performants que les autres, ne rencontreraient aucune difficulté, risquant peut-être de moins avoir l'opportunité de progresser ;

- d'autres élèves auraient l'occasion de «jouer» grâce à la résistance que leur offre une situation qui reste toutefois à leur portée, et d'apprendre par adaptation au milieu proposé ;

- d'autres encore, trop éloignés des outils nécessaires pour s'adapter à ce milieu, ne pourraient en bénéficier, et seraient alors contraints de dépendre de l'aide du maître ou d'accepter les réponses proposées par d'autres élèves.

Cette recherche s'est déroulée sur deux ans, elle porte sur des séances de lecture (découverte collective de texte). Quatre classes de cours préparatoire sont concernées la première année, les quatre enseignants sont expérimentés. La deuxième année, deux de ces enseignants sont observés face à une autre cohorte d'élèves de $\mathrm{CP}$, lors de séances de lecture.

\section{La Distance à la Performance Attendue, un indicateur du potentiel de l'élève face à la tâche prescrite par l'enseignant}

Les écrits de Vygotski (1997) permettent de comprendre que la zone proximale de développement de chaque élève explique des effets contrastés de l'efficacité de la médiation de l'enseignant. Cette médiation serait peu utile à un élève qui sait déjà exécuter la tâche proposée, elle serait féconde pour l'élève qui n'est pas trop éloigné des savoirs en jeu, elle risque de devenir inopérante pour un élève trop démuni face à la tâche.

Nous ne mesurons pas la zone proximale de développement de l'élève qui ne peut pas être accessible à partir d'une recherche focalisée sur l'apprentissage de la lecture au $\mathrm{CP}$, à partir de simples variables didactiques. Nous cherchons à étudier une dimension moins psychologique et plus contextualisée de l'activité de l'élève: sa Distance à la Performance Attendue (DPA). Il s'agit de l'écart entre ce qu'il sait faire et ce qu'il faudrait savoir faire pour effectuer la tâche proposée par l'enseignant; de l'écart entre 
ses performances potentielles et la performance attendue, révélée par l'analyse de la tâche que le maître a choisie. Toutefois, nos observations antérieures ont montré que de multiples interventions de l'enseignant, pendant le déroulement de la tâche, aident certains élèves à parcourir un chemin trop difficile pour eux. Ainsi, les performances des élèves à la tâche proposée pendant l'interaction risquent d'être significativement améliorées par l'accompagnement didactique de l'enseignant, par son rôle de médiateur, et par conséquent de ne pas révéler la distance effective de certains élèves aux performances attendues par le maître lorsqu'il choisit cette tâche. C'est pourquoi nous n'utiliserons pas les travaux des élèves réalisés en classe en réponse aux tâches proposées par l'enseignant.

Le déroulement d'une tâche est filmé à l'aide de deux caméras permettant de garder la trace de l'activité de l'enseignant et de ses élèves. Le jour même, ce film est étudié, il permet une analyse ergonomique de la tâche (difficultés spécifiques, savoirs en jeu, prérequis nécessaires, stratégies potentielles, etc.). Grâce à cette analyse de la tâche choisie par le maître, des tests sont élaborés : ils visent l'évaluation du " potentiel d'un sujet à qui on a prescrit cette tâche ». Un ou deux jours après la séquence filmée en classe, ces tests sont proposés aux élèves, en protocole individuel, en dehors du contexte classe. Cette méthodologie évalue, pour chaque élève, sa DPA, quantifiée de 0 à 1. Cette distance est nulle pour un élève qui se montre performant face aux tests issus de l'analyse de la tâche prescrite, elle est maximale pour l'élève qui ne réussit aucun de ces tests. Il s'agit d'une distance résiduelle, il peut y avoir eu apprentissage entre l'observation en classe et le moment de la mesure de la DPA, il reste cependant possible d'affirmer que si une distance est observée (le test révèle le faible potentiel d'un élève), alors c'est qu'elle était au moins aussi importante, pour cet élève, lorsque l'enseignant a proposé la tâche deux jours avant. Cette mesure est répétée plusieurs fois dans l'année : chaque séance observée donne lieu à la construction d'un test différent.

L'étude, réalisée pour le programme incitatif de recherche en éducation et formation (PIREF), est issue d'une recherche intitulée Caractérisation des pratiques d'enseignement et détermination de leur efficacité ; la lecture et les mathématiques au cours préparatoire (première primaire) (Sensevy, 2007).

Nous devons repérer les savoirs en jeu lors des séances de lecture, sans avoir l'ambition d'effectuer une analyse de l'activité cognitive de l'élève, ni d'accéder à ses stratégies (par exemple : s'appuie-t-il sur le contexte et le sens ou bien alors sur une dimension «mécanique » de la lecture ?).

Pour décrire le potentiel de chaque élève nous repérons, pour chaque tâche proposée, les outils de lecteurs nécessaires. Les analyses des tâches sont élaborées à partir des deux processus d'identification des mots : la voie directe et la voie indirecte :

- DPA Voie directe (ou adressage : reconnaissance des mots connus du lecteur par appariement direct de la configuration écrite du mot avec sa représentation en mémoire, phase orthographique sans recours à la conversion phonologique). Plusieurs mots isolés, issus du texte proposé par l'enseignant un ou deux jours avant, à lire en un temps qui ne laisse pas la possibilité d'utiliser la voie indirecte (sauf peut-être pour des déchiffreurs particulièrement véloces), ni l'appui sur le sens (absence de contexte) permettent d'obtenir la DPA voie directe ;

- DPA Voie indirecte (ou assemblage : traitement des mots inconnus du lecteur, par la transformation de l'information visuelle en information phonologique et par l'application de règles de correspondance entre graphies et phonies). La DPA voie indirecte est composée de trois types de DPA : 
- DPA lecture de syllabes : l'élève doit lire des syllabes isolées, observées dans la tâche proposée par l'enseignant.

- DPA lecture de sons isolés : il s'agit pour l'élève d'oraliser des graphèmes contenus dans le texte choisi par l'enseignant.

- DPA conscience phonologique : nous demandons aux élèves de décomposer certains mots du texte en phonèmes.

Nous rajoutons la DPA Voie mixte : l'élève doit lire des phrases équivalentes à celles étudiées lors de la séance de lecture. Il peut s'appuyer sur le sens; nous ignorons alors quelle stratégie il utilise.

Ainsi, après chaque séance de lecture observée, nous construisons un test qui mesure cinq types de DPA : DPA « voie directe », DPA «syllabes », DPA « sons », DPA « conscience phonologique » et DPA « voie mixte ».

Un enseignant choisissant un texte à faire découvrir à ses élèves, détermine des conditions différentes en fonction de leur potentiel. Les DPA spécifiques illustrent ces conditions différentes : certains élèves ont des DPA proches de $0:$ les outils nécessaires pour lire ce texte sont à leur portée; d'autres, proches de 1 , sont très démunis et dépendants face à ce même texte ${ }^{2}$.

Les relations entre DPA et apprentissage nécessitent des tests évaluant les progrès des élèves pendant l'année.

\section{Evaluation des progrès des élèves}

Nous avons, au cours de recherches antérieures, constaté que les évaluations utilisées dans le cadre scolaire pouvaient ne pas révéler certaines difficultés des élèves. Une de leurs limites s'explique par le fait que l'enseignant expérimenté connaît la forme, le contenu et les enjeux de ces évaluations. Elles peuvent donc implicitement "guider» sa pratique, certaines pratiques scolaires pourraient alors ressembler à une préparation au test et à sa spécificité, préparation limitant les difficultés effectives de l'épreuve. Nous avons donc utilisé deux tests standardisés : l'alouette et le E20 passés en début et en fin de CP. Il s'agit de tester chaque élève de la classe et de situer au mieux ses performances, à la fois sur le plan technique et sur le plan compréhension.

Le test de l'alouette (Lefavrais, 1967), utilisé depuis de nombreuses années par des psychologues scolaires, mesure uniquement la dimension " assemblage » du savoir lire de l'élève. Ce dernier n'a aucun recours possible au contexte (au sens du texte). Le test indique son âge lexique. Ainsi, par exemple, un test passé en septembre au CP peut indiquer que l'élève a un niveau « $\mathrm{CP}$ janvier » : nous considérons dans ce cas qu'il a quatre mois d'avance.

Le test du E20 (Khomsi, 1997), également utilisé par des psychologues scolaires, mesure le niveau de compréhension. Il s'agit, pour l'élève, d'identifier l'image parmi quatre correspondant à une phrase proposée.

\section{RESULTATS}

\section{Les élèves d'une même classe n'ont pas le même statut face aux tâches proposées par l'enseignant}

La DPA est un indicateur du statut de l'élève face à la tâche proposée. Après chaque séance observée, nous avons fait passer un test aux élèves, permettant d'obtenir pour chacun d'entre eux cinq types de DPA : voie directe, syllabes, sons, conscience phonologique, voie mixte. Les premiers résultats que nous présentons concernent la lecture de sons isolés (DPA « sons »). Les tests de la DPA prélèvent dans les textes 
choisis par les enseignant des graphèmes (ou, $u$, en, eau, ch, p, etc.). L'expérimentateur demande à chaque élève (protocole individuel, en dehors du contexte classe) de lire les graphèmes de ce test. Un score de zéro à un révèle une performance de l'élève, mais également les conséquences (pour l'élève concerné) d'un choix de niveau de tâche par l'enseignant. Ainsi, un ou deux jours après l'observation en classe, alors que l'enseignant est passé à l'étape suivante (a choisi un nouveau texte support d'apprentissage), la DPA donne à voir le potentiel de chaque élève face aux enjeux d'une situation d'apprentissage lui ayant été proposée.

Afin de simplifier la lecture de nos résultats nous avons choisi de les réduire en trois catégories (nulle, faible, forte ; choix arbitraire, mais qui permet de montrer des statuts d'élèves contrastés) :

- Les élèves ayant une DPA nulle : ce sont ceux qui maîtrisent toutes les lectures des graphèmes en jeu lors de la situation d'apprentissage (soit parce qu'ils les connaissaient déjà, soit parce que la situation proposée a consolidé cet apprentissage) ;

- Les élèves ayant une DPA faible (supérieure à 0 et inférieure à 0,4 ) : certaines graphies sont encore inconnues ;

- Les élèves ayant une DPA forte (supérieure ou égale à 0,4 ) : le déficit instrumental de ces élèves est notable, voire important. Nous pouvons considérer, d'une part, que la situation d'apprentissage qui leur a été proposée comportait un nombre significatif de graphies qu'ils ne connaissaient pas, d'autre part que cette situation n'a pas permis de réduire de façon significative ce déficit.

\begin{tabular}{|c|c|c|c|c|c|c|c|c|c|c|c|c|}
\hline \multirow{3}{*}{$\begin{array}{c}\text { DPA } \\
\text { « Sons » }\end{array}$} & \multicolumn{12}{|c|}{ Classes } \\
\hline & \multicolumn{4}{|c|}{ Décembre } & \multicolumn{4}{|c|}{ Mars } & \multicolumn{4}{|c|}{ Juin } \\
\hline & 1 & 2 & 3 & 4 & 1 & 2 & 3 & 4 & 1 & 2 & 3 & 4 \\
\hline Nulle & 7,6 & 43,7 & 0 & 15,3 & 33,3 & 16,6 & 65 & 9,5 & 61,1 & 66,7 & 66,6 & 54,5 \\
\hline Faible & 46,2 & 25 & 75 & 38,5 & 38,1 & 55,6 & 25 & 66,7 & 27,8 & 11,1 & 27,8 & 36,4 \\
\hline Forte & 46,2 & 31,3 & 25 & 46,2 & 28,6 & 27,8 & 10 & 23,8 & 11,1 & 22,2 & 5,6 & 9,1 \\
\hline
\end{tabular}

\section{Tableau I. - Pourcentage d'élèves ayant une DPA « sons » nulle/faible/forte en fonction des classes et de la période}

Nous proposons d'analyser les pourcentages d'élèves ayant une DPA forte. Leurs chances d'accéder au sens du texte en étaient d'autant plus réduites ${ }^{3}$. Ce tableau de résultats montre que dans toutes les classes observées, le fait de choisir un texte support d'apprentissage provoque une situation inéquitable. Dans cet exemple, l'inéquité est repérée sur une variable didactique «de base»: les graphies des phonèmes. C'est une façon de montrer à la fois l'obstacle technique qu'un enseignant affronte face à un collectif hétérogène et des opportunités d'apprendre très contrastées. Les méthodes d'enseignement, ou les compétences des enseignants ne sont pas en cause face à ce constat de choix de support d'apprentissage. Quel que soit le choix, il conviendra mieux à certains élèves qu'à d'autres.

Ces premiers résultats montrent que le pourcentage d'élèves à forte DPA « sons » diminuent d'une période à l'autre. En considérant toutes classes confondues $37 \%$ des élèves ont une forte DPA en décembre ; $22,5 \%$ en mars ; $12 \%$ en juin. Concernant la connaissance des sons en jeu dans les tâches proposées, le déficit instrumental de certains élèves est constant alors que par ailleurs les évaluations 
standardisées montrent qu'ils ont progressé. Malgré leur progression, l'avancée didactique de la classe les maintient à l'écart: une forme d'exclusion à un niveau instrumental qui aura très probablement des conséquences sur la suite des apprentissages.

L'analyse des autres DPA (lecture de syllabes, voie mixte, voie directe) est comparable à celle que nous venons de présenter. Lorsque la variable didactique est plus complexe, le nombre d'élèves ayant une forte DPA augmente. Par exemple pour la DPA « voie mixte » $41 \%$ des élèves ont une forte DPA en décembre ; $32 \%$ en mars ; $23 \%$ en juin.

La seule observation des séances de lecture ne permet pas toujours de faire le constat du "passage à l'étape suivante alors que tous les élèves n'ont pas appris ». En effet, les élèves les plus faibles bénéficient de la médiation de l'enseignant, ils obtiennent les réponses aux questions posées. Suite à un travail d'étayage, à des procédures apparentées à de la pédagogie différenciée, ils peuvent redire le contenu du texte proposé.

Goigoux et Thomazet (1999) avaient déjà repéré que les enseignants, afin de pallier les difficultés d'élèves de SEGPA ${ }^{4}$, les aidaient fréquemment. Ces derniers, n'ayant pas conscience de cette aide, s'avéraient incapables de réutiliser seuls les procédures mises en œuvre. Bautier et Rochex (2004) évoquent le contrôle de l'activité des élèves en difficulté grâce à des tâches tellement simplifiées qu'elles ne font appel qu'à des traitements de bas niveau.

De notre côté, nous constatons que la performance obtenue légitime le fait de pouvoir passer à l'étape suivante (aussi bien aux yeux de l'observateur qu'à ceux de l'enseignant : l'évaluation à court terme, proche du contexte de la situation didactique participe à cette légitimation). Cependant, un ou deux jours après, la mesure de la DPA montre que l'obtention de la réponse n'a pas suffi pour apprendre. Pour certains élèves trop éloignés des savoirs en jeu, les aides, médiations de l'adulte et étayages divers contribueraient, à court terme, à masquer leur exclusion. Ces procédures d'aide seraient le complément indispensable des contraintes liées au choix des tâches; elles sont probablement intégrées sous forme de schèmes professionnels.

Les mesures de DPA contribuent ainsi à montrer que le contrat didactique n'est pas identique pour l'ensemble des élèves de la classe. A l'instar de Schubauer-Leoni (1991), nous pouvons évoquer des " contrats didactiques différentiels» : les attentes implicites des enseignants sont différentes en fonction du statut des élèves, et ces derniers, par leur activité, montrent qu'ils perçoivent ces différences d'attentes.

Nous avons voulu observer de façon plus précise la relation entre ces DPA observées et les résultats des élèves en lecture. Pour ce faire, nous avons dû utiliser une méthodologie plus " lourde » nous imposant de réduire l'étude à deux classes, pendant une deuxième année scolaire. Nous avons choisi d'étudier les deux classes situées dans des écoles où les conditions d'apprentissage semblent les plus favorables (ces deux écoles ont significativement moins d'élèves en difficulté que les deux autres). En effet, notre objectif consiste à décrire et à identifier la complexité des conditions de travail de l'enseignant et de ses élèves, même lorsque le contexte de l'école n'est pas défavorable. La deuxième année, deux des enseignants sont donc observés face à une autre cohorte d'élèves de CP. 


\section{Des classes encore plus hétérogènes en fin d'année}

Afin de donner une image concrète de l'hétérogénéité des classes, nous proposons des données traduites en «mois d'avance ou de retard " pour la dimension technique (Alouette) et par une note sur vingt pour la dimension compréhension (E20).

La prise en compte de ces deux dimensions permet de répartir, pour chaque classe, les élèves en trois groupes comportant le même nombre d'élèves : les bons lecteurs, les lecteurs moyens, les faibles lecteurs ${ }^{5}$.

N.B. : on ne peut pas dire que les élèves moyens en décembre sont les mêmes que les élèves moyens en juin (la suite de l'étude apportera des précisions). Les doubles flèches indiquent des différences significatives.

\begin{tabular}{|l|c|c|c|c|c|c|c|}
\cline { 2 - 7 } & \multicolumn{3}{c|}{ Niveau technique } & \multicolumn{4}{c|}{ Niveau compréhension } \\
\cline { 2 - 7 } & \multicolumn{3}{c|}{ (en mois d'avance ou de retard) } & \multicolumn{4}{c|}{ (note sur 20) } \\
\cline { 2 - 7 } & Bons lecteurs & Lecteurs moyens & Faibles lecteurs & Bons lecteurs & Lecteurs moyens & Faibles lecteurs \\
\hline Classe 2 & 1,1 & 0,7 & $\rightarrow$ & -1 \\
\hline Classe 3 & 4,7 & 1,1 & $\rightarrow$ & $-1,8$ \\
\hline
\end{tabular}

Tableau II. - Performances relevées en décembre

Nos deux classes sont, au niveau de la performance moyenne, équivalentes en décembre ( $\mathrm{T}$ de Student, technique : $\mathrm{p}=.26$; compréhension: $\mathrm{p}=.20$ ), mais leur hétérogénéité, dès le début décembre, est déjà bien visible. On constate dans la classe trois un groupe d'élèves ayant, en moyenne, presque cinq mois d'avance, quand le groupe des plus faibles a presque deux mois de retard.

\begin{tabular}{|c|c|c|c|c|c|c|}
\hline & \multicolumn{3}{|c|}{ Niveau technique } & \multicolumn{3}{|c|}{ Niveau compréhension } \\
\hline & \multicolumn{3}{|c|}{ (en mois d'avance ou de retard) } & \multicolumn{3}{|c|}{ (note sur 20) } \\
\hline & Bons lecteurs & Lecteurs moyens & Faibles lecteurs & Bons lecteurs & Lecteurs moyens & Faibles lecteurs \\
\hline Classe 2 & 7 & 0,6 & $-3,8$ & 15,4 & 13,6 & 9,8 \\
\hline Classe 3 & 7,6 & $-1,3$ & $-4,3$ & 15,1 & 14,6 & 10,7 \\
\hline
\end{tabular}

\section{Tableau III. - Performances relevées en juin}

En juin, les moyennes des deux classes ne sont pas significativement différentes (technique : $\mathrm{p}=.63$; compréhension $: \mathrm{p}=.39$ ).

La comparaison de ces deux tableaux montre, dans les deux classes, que les écarts se sont creusés, mais ces comparaisons décembre / juin ont un sens particulier dû à la nature des tests utilisés.

Le test technique (Alouette) est standardisé de façon à suivre l'évolution « normale » d'un lecteur et indique «l'âge lexique ». Ainsi, un sujet qui avait une place sur l'échelle du test en décembre (un mois de retard par exemple) pourrait, selon la normalisation sur laquelle est fondé le test, avoir conservé ce mois de retard en juin. Dans ce cas-là, c'est la loi des grands nombres, bien que discutable, qui détermine cet " âge lexique », de façon statistique, après observation des performances d'un grand nombre d'élèves apprenant à lire. Ce n'est pas cette norme qui nous intéresse, mais plutôt les écarts à la norme; les parcours différenciés, contrastés, des élèves d'une même classe ; des « effets $\mathrm{CP}$ » contrastés en fonction du statut de l'élève. En fin de CP, les bons lecteurs ont plus de sept mois d'avance (de décembre à juin, ils ont progressé par rapport à leur niveau initial); les moyens oscillent autour du niveau attendu à cette 
période-là. Quant aux faibles lecteurs, ils ont très peu bénéficié de leur $\mathrm{CP}$ (autour de quatre mois de retard en fin d'année). L'écart entre les plus performants et les plus faibles est en moyenne de onze mois, soit près d'une année. Rappelons que ces deux classes, de centre ville, ne travaillent pas dans des conditions difficiles, mais n'échappent pas à cette forte hétérogénéité. Des enseignants travaillant dans un milieu réputé moins difficile que d'autres se trouvent toutefois face à un collectif dont les besoins sont fortement contrastés: une contrainte qui pèse sur les choix et leurs conséquences, etc., qui pèse sur la construction de l'expérience de l'enseignant, dès ses premiers pas dans le métier.

Le test compréhension (E20) est une épreuve identique en décembre et en juin. Seule différence: il s'agit de compréhension de l'oral en décembre, et de la compréhension des mêmes phrases à l'écrit en juin. Malgré l'effet « retest », qui devrait favoriser la performance à la deuxième épreuve, des élèves vont obtenir en juin une performance plus faible ( $\mathrm{Z}$ de $\left.\mathrm{Wilcoxon}^{6} ; \mathrm{p}=.0002\right)$. La dimension technique de la lecture reste encore une "tâche ajoutée » qui, contrairement à un automatisme, a un coût cognitif provoquant une performance en compréhension inférieure à celle mesurée 9 mois avant. En compréhension le groupe des faibles est celui qui est le plus loin de ses performances initiales à l'oral.

Connaissant l'évolution des performances de chaque élève dans l'année, nous pouvons revenir sur les conditions dans lesquelles il était placé, à chaque période de l'année, face à la tâche que proposait l'enseignant, grâce à la mesure de la Distance à la Performance Attendue (DPA).

\section{Des apprentissages contrastés au sein d'une même classe}

Le graphe bivarié (corrélation), placé en annexe, repose sur les performances au test de l'alouette (performances techniques, d'assemblage). Il résume les différents parcours des élèves de ces deux $\mathrm{CP}$.

Ici, les catégories de lecteurs bons/moyens/faibles ne sont pas obtenues en divisant la classe en trois groupes d'élèves de même taille, mais en se basant sur leur âge lexique (score au test de l'alouette) : les élèves considérés comme faibles sont ceux qui ont plus d'un mois de retard en début d'année, les bons lecteurs ont plus d'un mois d'avance. Cette catégorisation permet maintenant de tenir compte du niveau «objectif» des élèves, et non de leur niveau relativement au groupe-classe :

- parmi les huit élèves faibles en début d'année, sept se retrouvent dans la même catégorie en fin d'année ;

- les neuf élèves performants en début d'année le sont encore en fin d'année ;

- parmi les vingt neuf élèves moyens en début de $\mathrm{CP}$, vingt quatre changent de statut en fin de CP : certains sont intégrés dans le groupe des bons lecteurs alors que d'autres se retrouvent dans le groupe des faibles lecteurs.

Ce graphe en annexe montre que les élèves faibles et forts en début d'année gardent leurs statuts respectifs en fin d'année, tandis que les élèves moyens en début d'année sont ceux qui changent le plus de statut. Un chi2 croisant le statut de l'élève en début d'année (moyen/autre) avec la variation de performance en cours d'année (faible/forte, c'est-à-dire inférieure ou non à deux mois d'âge lexique) confirme en effet cette interprétation de la représentation graphique $(\mathrm{p}=.04)$.

Ces résultats, qui n'apparaissent pas lorsque l'on traite de progrès globaux des classes, vont dans le sens d'un « effet maître » et/ou d'un « effet CP », bénéficiant à une partie des élèves moyens. Pour ce qui est de la dimension technique de la lecture, les progrès différentiels montrent ainsi que la classe de $\mathrm{CP}$ comporte peu d'enjeux pour les 
élèves performants, que leurs compétences initiales assurent de ne pas changer de statut, et peu d'enjeux pour les élèves faibles, qui n'auront que de rares occasions de rattraper leur retard initial (ce qui ne signifie pas que ces deux catégories d'élèves ne font pas de progrès). Ce constat, concernant les élèves les plus faibles qui ne peuvent rattraper leur retard initial, nous conduit à affirmer qu'il est urgent de s'interroger sur ce « retard initial » à l'entrée au CP.

A l'inverse, l'enjeu est important pour les élèves moyens, qui peuvent en fin d'année avoir un statut d'élève faible ou fort.

Sachant comment chaque élève "moyen en décembre » a terminé l'année, il faut maintenant tenter de savoir à partir de quelle période de l'année nos DPA permettaient de prévoir le sort des moyens qui progressent plus ou moins pendant l'année.

\section{Des opportunités d'apprendre très contrastées au sein d'une même classe}

En décembre, les DPA des moyens qui progresseront le plus dans l'année ne sont pas significativement différentes de celles des moyens qui progresseront le moins ( $U$ de Mann Whitney pour chaque classe ${ }^{7}$ ). Cela confirme que les élèves moyens en début d'année ont un potentiel équivalent face aux tâches proposées par les enseignants.

Or, dès le mois de mars, les DPA des élèves moyens qui progressent sont significativement plus faibles (classe $2: p=.01$; classe $3: p=.02$ ). Ce résultat montre que, dès mars, les choix de l'enseignant restent proches du potentiel des élèves moyens qui progressent : les tâches choisies deviennent significativement plus difficiles pour les élèves moyens qui décrochent (d'autant plus difficiles pour les élèves faibles dès le début de l'année). Face à ces opportunités d'apprendre le groupe des moyens s'est divisé. On ne peut pas chercher des raisons d'échec en invoquant un retard « technique » initial. On pourrait faire l'hypothèse que des lacunes en compréhension les empêchent de progresser en assemblage autant que les autres élèves moyens, or ce n'est pas non plus le cas (chi2; $\mathrm{p}=.93$ ). Des solutions pédagogiques ou didactiques méritent d'être étudiées, c'est le groupe d'élèves dans la classe qui semble être le plus sensible à un effet école, à un effet CP, à un effet maître. L'analyse des DPA permet de constater un ajustement des choix de l'enseignant à une période où quelques mois de $\mathrm{CP}$ ont modifié le statut de quelques élèves.

En résumé, les élèves progressent au cours de l'année ; le curseur du niveau de difficulté des tâches progresse également. Mais, cette augmentation n'est pas aléatoire : elle est majoritairement «calée » sur le potentiel des mêmes élèves (ici les élèves moyens qui progressent). Ces élèves pourraient correspondre au groupe de pilotage («steering group») mis en avant par Dahllöf (1967): ce groupe d'élèves-repères jouerait un rôle important notamment lorsque le temps disponible n'est pas suffisant pour permettre à l'ensemble des élèves d'atteindre un but fixé ; dans ce cas, l'enseignant se baserait sur les besoins du groupe de pilotage pour prendre ses décisions. Il serait donc cohérent que les progrès de ce groupe soient plus importants que ceux des autres élèves : le même support d'apprentissage (qui n'est pas un support construit pour évaluer les élèves) provoquerait des opportunités d'apprendre très contrastées en fonction du statut de l'élève.

\section{ENSEIgNER FACE A UN COLLECTIF HETEROgENE, UN OBSTACLE TECHNI QUE}

Il est, tout d'abord, utile de préciser que nos enseignants ne sont pas des enseignants maladroits, ils sont expérimentés, leurs responsables institutionnels et nos observations le confirment. Ils enseignent (classes deux et trois étudiées sur deux ans) 
dans un milieu qui, en aucun cas, ne peut être considéré comme défavorisé. Ils choisissent quotidiennement les tâches qu'ils proposent à leurs élèves : ce sont des supports d'apprentissage (par exemple un texte à lire). Ce choix est délicat car il concerne un collectif hétérogène à tel point que, quelle que soit leur décision de choix du niveau de difficulté, ils semblent ne pouvoir jouer que sur la répartition de leurs élèves selon trois statuts :

1. l'élève qui sait déjà presque tout faire ;

2. l'élève qui sait suffisamment faire : l'énigme didactique proposée provoque le juste déséquilibre favorisant l'apprentissage ;

3. l'élève trop démuni pour lequel l'énigme est probablement située au delà de sa zone proximale de développement.

Partant de ce constat d'une diversité de maîtrise de la lecture en fin de CP pour les élèves «moyens » de début d'année, Clanet (2007), à partir de l'analyse d'une de nos classes, en vient à constater que « la fréquence des interactions qui se nouent entre le maître et ces élèves moyens est en lien avec le degré de maîtrise de la lecture en fin de C.P.; ceux qui sont, soit destinataires, soit à l'origine de l'action verbale et qui reçoivent davantage d'informations et d'encouragements positifs sont ceux des moyens du début d'année qui se retrouvent bons lecteurs en fin d'année. A l'inverse ceux qui sont moins fréquemment engagés dans les actions-interactions ne connaîtront pas cette progression et au contraire se retrouveront être en difficulté pour lire en fin d'année ${ }^{8} »$. Nous avons là un des éléments potentiellement explicatifs de ce différentiel de réussite, la médiation que met en place l'enseignant diffère suivant les élèves.

Nos hypothèses explicatives peuvent aussi s'appuyer sur des résultats antérieurs (Maurice, 1996a) qui montraient que l'enseignant, à la lecture des performances d'un seul élève, modifiait, de façon pertinente, ses prédictions sur le travail d'autres élèves de la classe, sans avoir eu besoin d'observer ce qu'ils avaient effectivement fait. L'économie cognitive, étroitement liée à l'évolution de l'expérience de tout professionnel, expliquerait que l'enseignant utilise des prises d'informations échantillonnales car il lui est impossible de prendre en compte l'exhaustivité des informations disponibles. Il connaît des élèves en fonction d'autres élèves repères, il regroupe certains élèves en fonction de critères probablement liés à leur potentiel face à des tâches scolaires qui ont une forte valeur d'utilité pour piloter la classe, mais, de plus, il est peut-être aussi contraint de cibler spécifiquement ses actions sur certains élèves pour faire évoluer l'ensemble de la classe. Il serait obligé de « faire avec » une hiérarchie de potentiels; le groupe des moyens qui progressent serait le centre de gravité d'une classe très hétérogène, le lieu où ses choix (niveau de difficulté des tâches et aides qui en découlent) conviennent «au mieux» à ce groupe tout en restant supportables par les autres. Nos résultats s'expliqueraient en partie par la nécessité d'élever au maximum ce centre de gravité : cela permettrait de faire progresser tous les élèves tout en conservant le contrôle de la classe, sans pour autant modifier les hiérarchies inter élèves (avec, selon nos résultats, le risque de les renforcer).

La DPA participerait à la modélisation de schèmes professionnels constitués d'anticipations sur l'activité des élèves, de prises d'informations et régulations en cours de séance... Ces schèmes seraient construits en réponse à des obstacles techniques liés à l'enseignement face à un groupe hétérogène. La DPA aiderait également à expliquer, au sein d'une même classe, des opportunités d'apprendre contrastées.

Ainsi, nos observations mettent en évidence une image de l'effet de la "machine enseignement face à un collectif très hétérogène ». La marge de manœuvre de l'enseignant serait limitée par de fortes contraintes qui constitueraient pour lui un 
véritable «obstacle technique ». Nos choix méthodologiques, s'appuyant sur des statistiques, négligent tout ce que l'enseignant fait pour lutter contre ces contraintes, mais ils révèlent le mécanisme qu'il affronte au quotidien.

La pédagogie différenciée et les médiations de l'adulte sont utiles lorsqu'elles permettent à certains élèves d'accéder à des performances auxquelles ils n'auraient pu accéder seuls. Cependant, à partir d'un certain seuil, augmenter l'aide nécessaire à un élève (jusqu'à lui donner la bonne réponse) aurait pour effet de réviser à la baisse son activité cognitive, de masquer son exclusion, de justifier le passage à l'étape suivante. La DPA révèle, à ce moment précis de l'avancée didactique, que les procédures de type "pédagogie différenciée » ont de sérieuses limites. L'enseignement face à un collectif hétérogène produit "mécaniquement» une exclusion de certains élèves. Il semble délicat d'attribuer ce fait à d'éventuelles défaillances professionnelles des enseignants. Des solutions restent donc à « inventer».

La mise en place des cycles a tenté de pallier ce problème en préconisant d'étaler l'apprentissage de la lecture sur trois ans, mais nos résultats permettent d'entrevoir quelques obstacles à cette prescription :

- L'hétérogénéité déjà observable en début d'année va augmenter de façon très significative : dès le démarrage d'un enseignement systématique de la lecture, des élèves très rapides accentuent l'écart initial ;

- Certains élèves ne sont pas «prêts » à affronter cet enseignement systématique qui impose un rythme qu'ils ne pourront pas supporter, alors que leur date de naissance est le critère de leur présence dans ce système qui va les exclure ;

- Le bon fonctionnement du groupe classe dépend largement du fait que les élèves soient mobilisés, occupés, travaillent, etc. Cet enrôlement dépendrait du pouvoir de mobilisation des tâches proposées. Or, lorsqu'un certain pourcentage d'élèves ont épuisé l'énigme didactique, ces élèves qui «attendent » menacent la légitimité de l'enseignant, celui-ci prend le risque de perdre le contrôle du groupe : il est alors contraint à proposer des tâches qui renouvellent l'énigme (cf. Maurice, 2006b) ;

- L'hétérogénéité est telle qu'elle menacerait les limites matérielles et cognitives de tout être humain devant affronter cette complexité. Une telle affirmation permettrait de comprendre les limites des prescriptions de type «pédagogie différenciée » (cf. Maurice, 2006a);

- L'enseignant débutant affronte ces contraintes dès ses premiers pas dans le métier. Face à elles il développe des habiletés cognitives, des savoir faire professionnels qui échappent aux institutions de formation, s'apparentent à des schèmes professionnels efficaces (choix d'un niveau de difficulté de tâche par exemple). Ces savoir faire sont très opérationnels dans l'action, mais pas toujours accessibles dans les moments de réflexion sur l'action.

Toutes ces contraintes convergent pour inciter les enseignants à apprendre à proposer des tâches acceptables par le collectif. L'aide aux élèves les plus démunis masque, à court terme, le fait qu'ils ne peuvent pas suivre et justifie de "passer à l'étape suivante». Ce processus participerait à expliquer l'augmentation de l'hétérogénéité et la grande difficulté de prise en compte des rythmes très différents des élèves d'une même classe. Il ne serait pas dû à des défaillances professionnelles, il serait impossible pour un enseignant, au cours préparatoire, d'assumer une hétérogénéité au delà d'un certain seuil, sans creuser les écarts et laisser des élèves démunis. On risque ainsi de refuser, à juste titre, des ghettos formels, mais de ne pas 
éviter de placer certains élèves dans des situations significativement moins favorables pour apprendre.

\section{PAS DE CONCLUSIONS HATIVES}

- Les obstacles évoqués ci-dessus ne sont pas liés à un problème de "méthode " d'enseignement de la lecture : un enseignant qui focaliserait son action uniquement sur la dimension « assemblage » n'échapperait pas à l'émergence de forts contrastes entre les progrès des élèves et à l'impérieuse nécessité de proposer alors des tâches acceptables par le collectif, au risque de perdre le contrôle de sa classe.

- Les aides (ponctuelles) apportées par le réseau d'aides ou organisées sous forme de PPRE $^{9}$ par exemple sont indispensables, elles réduisent les difficultés des élèves. Cependant, en ce qui concerne les élèves en difficulté, le temps d'exposition annuel à des situations bien trop difficiles est tellement plus long que celui de l'aide individualisée qu'il est important de tenter des solutions concernant l'enseignement collectif.

- Nos résultats ne concernent que l'apprentissage de la lecture au CP. Il est impossible de les généraliser à des «distances au problème posé à un groupe d'élèves »".

- Ce travail, imposant une méthodologie très lourde, n'a pas pu être réalisé sur un nombre suffisant de classes pour permettre une généralisation incontestable. Des études complémentaires devront être réalisées.

Nos résultats pourraient paraître trop pessimistes. C'est dû au fait qu'ils s'appuient sur des procédures statistiques qui déshumanisent les contextes observés pour mettre en évidence un mécanisme, un obstacle professionnel que nos enseignants doivent affronter au quotidien. L'identification de ce type d'obstacle permet d'éviter :

- des explications dérivant trop facilement vers des carences de la compétence professionnelle ;

- des conseils bien faciles à formuler, mais bien difficiles à mettre en œuvre.

Grâce à la qualité de leur relation aux élèves, à leur empathie, à leur inventivité, etc., les enseignants peuvent très certainement limiter les effets de cet obstacle. D'ailleurs, ces dimensions humaines, indispensables à un geste professionnel efficace, risquent bien d'expliquer des différences interindividuelles, mais elles ne peuvent, à elles seules, lutter contre un tel obstacle. Des solutions doivent être "inventées », en grande partie sur le terrain des pratiques, grâce à la collaboration des enseignants. Il faudrait alors oser avouer qu'à l'heure actuelle il existe très peu de solutions opérationnelles: seul moyen pour permettre aux praticiens de passer du statut « d'accusé contraint de se protéger » à celui de collaborateur inventif.

$$
\begin{aligned}
& \text { Jean-Jacques Maurice } \\
& \text { jmaurice@univ-tlse2.fr }
\end{aligned}
$$

Centre de recherches en éducation, formation et insertion, université de Toulouse

Audrey Murillo

murillo@univ-tlse2.fr

Centre de recherches en éducation, formation et insertion, université de Toulouse

\section{BIBLIOGRAPHIE}

AMIGUES R. (2003). «Pour une approche ergonomique de l'activité enseignante ». Skholê, Hors-série 1, p. 5-16. 
BAUTIER E. \& ROCHEX J.-Y. (2004). «Activité conjointe ne signifie pas significations partagées ", in C. Moro et R. Rickenmann (Ed.), Situation éducative et signification. Bruxelles : De Boeck.

BRESSOUX P., BRU M., ALTET M. \& LAMBERT C. (1999). "Diversité des pratiques d'enseignement à l'école élémentaire ». Revue française de pédagogie, $\mathrm{n}^{\circ} 126$, p. 97-110.

BROUSSEAU G. (1986). «Fondements et méthodes de la didactique des

mathématiques ». Recherches en Didactique des Mathématiques, vol.7, n², p.33-115.

CLANET J. (2007). «Eléments organisateurs de séances de lecture en cours préparatoire ». Repères, $\mathrm{n}^{\circ} 36$, p. 211-230.

DAHLLOF U. (1967). Ability grouping and the teacher process. Stockholm : Almqvist et Wiksell.

DOYLE W. (1985). «Recent research on classroom management: implications for teacher preparation ». Journal of Teacher Éducation, vol. XXXVI, n³, p.31-35.

GOIGOUX R. \& THOMAZET S. (1999). «Pratiques et Conceptions de l'enseignement de la lecture dans l'enseignement adapté ». Les actes de lecture, $\mathrm{n}^{\circ}$ 67, p. 53-62.

GENTAZ E. \& DESSUS P. (Ed.). (2004). Comprendre les apprentissages. sciences cognitives et éducation. Paris : Dunod.

KHOMSI A. (1997). Évaluation des compétences en lecture, E20. Paris : Editions du centre de psychologie appliquée.

LEFAVRAIS P. (1967). Test de l'alouette. Paris : ECPA.

LEPLAT J. \& HOC Jean-Michel. (1983). «Tâche et activité dans l'analyse psychologique des situations ». Cahiers de Psychologie Cognitive, vol. III, $\mathrm{n}^{\circ} 1$, p. 49-63.

LUNDGREN U. (1972). Frame factors and the teacher process. Stockholm : Almqvist et Wiksell.

MAURICE J.-J. (1996a). «Une connaissance de l'élève dépendante des contraintes de l'action ». Revue française de pédagogie, $\mathrm{n}^{\circ} 114$, p. 85-96.

MAURICE J.-J. (1996b). «Problèmes multiplicatifs: l'expérience de l'enseignant, l'action effective de l'élève ». Recherches en Didactique des Mathématiques, vol.16, $\mathrm{n}^{\circ} 3$, p. 323-348.

MAURICE J.-J. (2006). "L'expérience de l'enseignant: une réflexivité limitée ». Revue des hautes écoles pédagogiques et institutions assimilées de Suisse romande: formations et pratiques d'enseignement en questions, $\mathrm{n}^{\circ} 3$, p. 53-67.

MAURICE J.-J. «Des contraintes du métier d'enseignant participent-elles à la construction de son expérience? ». In E. Gentaz, P., Dessus (Ed.), Apprendre et enseigner à l'école : sciences cognitives et éducation. Paris : Dunod. p. 219-234.

MURILlO A. (2004). La Distance à la Performance Attendue: un indicateur pour l'étude des interactions enseignants-élèves médiatisées par la tâche scolaire. Mémoire de DEA non publié, sciences de l'éducation, Toulouse le Mirail, GPECREFI.

RABARDEL P. (1995). Les hommes et les technologies: approche cognitive des instruments contemporains. Paris : Armand Colin.

SARRAZY B. (2003). "Les hétérogénéités dans l'enseignement des mathématiques ». Éducational Studies in Mathematics, $\mathrm{n}^{\circ} 49, \mathrm{p} .89-117$.

SCHUBAUER-LEONI M.-L. (1991). «L'évaluation didactique : une affaire contractuelle. » In J. Weiss (ed.) L'évaluation : problème de communication. Cousset et Neuchâtel : DelVal et IRDP. p. 79-95. 
SENSEVY G. (Dir.) (2007). Caractérisation des pratiques d'enseignement et détermination de leur efficacité. La lecture et les mathématiques au cours préparatoire (Première primaire). Rapport au Programme Incitatif de Recherche en Éducation et Formation.

VERGNAUD G. (1985). "Concepts et schèmes dans une théorie opératoire de la représentation ». Psychologie française, vol. 30, n 3-4, p. 245-252.

VYGOTSKI L. (1997). Pensée et langage. Paris : La Dispute. 
ANNEXE

Des élèves bons lecteurs en décembre resteront bons lecteurs en juin

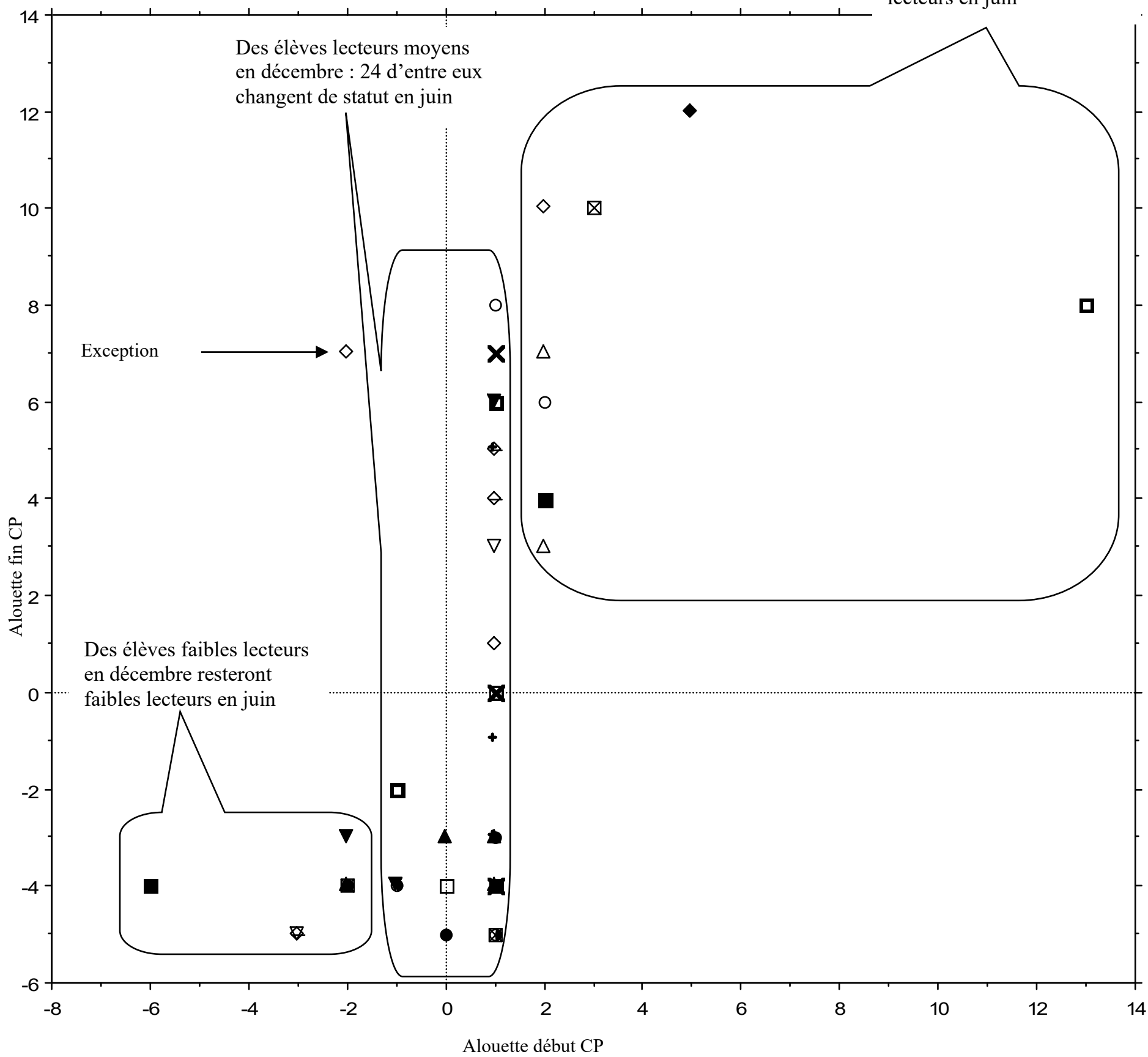

Parcours des élèves pour la dimension technique de la lecture

Chaque élève est représenté par un point: en abscisse, ses performances techniques en début de $\mathrm{CP}$, en ordonnée ses performances techniques en fin de $\mathrm{CP}^{10}$.

${ }^{1}$ Cf. Tâche/activité : Leplat et Hoc, 1983. En prescrivant une tâche l'enseignant attend une activité de l'élève (la mise en œuvre de savoirs ou savoir faire en jeu dans cette tâche). Cependant, l'activité effective de certains élèves, en partie inobservable, est parfois différente de celle attendue par l'enseignant. Notre objectif consiste à tenter d'approcher, grâce à un test, l'activité effective de chaque élève.

${ }^{2}$ La mesure est élaborée à partir du nombre d'erreurs. Exemple de test pour évaluer la DPA « lecture de syllabes »: nous proposons dix syllabes à lire (syllabes observées dans la tâche proposée par l'enseignant). L'élève qui sait lire 
ces dix syllabes obtient une DPA égale à 0 ; celui qui n'en lit aucune a une DPA égale à 1 ; celui qui en lit deux à une DPA de 0,2 ..

${ }^{3}$ Sans pour autant affirmer que la lecture des phonèmes est « la » clé de l'accès au sens, en effet les autres DPA non présentées en détail dans ce texte (voie directe, voie mixte, lecture de syllabes) apportent d'autres dimensions de la lecture et confirment la distance de ces mêmes élèves aux tâches proposées

${ }^{4}$ Sections d'enseignements généraux et professionnels adaptés

${ }^{5}$ Cette méthodologie permet de ne pas « choisir » un seuil distinguant différents niveaux de lecteurs et de ne pas appliquer ce même choix sur deux classes différentes. La médiane aurait pu être une solution, mais elle dichotomise la classe, méthode qui s'approche du tout ou rien et a peu de chances de représenter la réalité d'une classe. Partager en trois groupes nous a semblé plus cohérent avec ce que disent habituellement les enseignants lorsqu'ils parlent de leurs élèves.

${ }^{6}$ Ce test permet de comparer deux groupes appariés (avec indication du seuil de prise de risque $: \mathrm{p}=.01$ signifiant qu'on a moins d'une chance sur 100 de se tromper en affirmant qu'un groupe réussit mieux qu'un autre).

${ }^{7}$ Toutes DPA confondues. Ce test permet de savoir si un groupe est plus performant qu'un autre (avec indication du seuil de prise de risques).

${ }^{8}$ Sur tous ces aspects $\mathrm{p}<.05$.

${ }^{9}$ Programme Personnalisé de Réussite Educative

${ }^{10}$ Un élève dont les coordonnées sont $(0,0)$ est à un niveau normal en début de $\mathrm{CP}$ et en fin de $\mathrm{CP}$. Un élève dont les coordonnées sont $(0,4)$ est un élève qui a un niveau normal en début de $\mathrm{CP}$, et qui a quatre mois d'avance en fin de CP. 